\title{
Blind sectional image reconstruction for optical scanning holography
}

\author{
Xin Zhang, ${ }^{1}$ Edmund Y. Lam, ${ }^{1, *}$ Taegeun Kim, ${ }^{2}$ You Seok Kim, ${ }^{2}$ and Ting-Chung Poon ${ }^{3}$ \\ ${ }^{1}$ Imaging Systems Laboratory, Department of Electrical and Electronic Engineering, The University of Hong Kong, \\ Pokfulam Road, Hong Kong \\ ${ }^{2}$ Department of Optical Engineering, Sejong University, 98 Kunja-dong, Kwangjin-gu, Seoul 134-747, South Korea \\ ${ }^{3}$ Bradley Department of Electrical and Computer Engineering, Virginia Tech, \\ Blacksburg, Virginia 24061, USA \\ *Corresponding author: elam@eee.hku.hk
}

Received June 1, 2009; revised September 10, 2009; accepted September 10, 2009; posted September 16, 2009 (Doc. ID 112095); published October 6, 2009

Optical scanning holography is a powerful holographic recording technique in which only a single twodimensional scan is needed to record three-dimensional information. As in standard digital holography, for the reconstruction of a sectional image, the resulting data must then be postprocessed to obtain sectional content. We propose a blind sectional image reconstruction technique to automate the data processing. This reconstruction uses edge information to determine the appropriate Fresnel zone plates automatically and applies inverse imaging to recover the sectional images with significant suppression of the defocus noise. The experimental data used to verify the algorithm are measured from a physical implementation of the optical scanning holography system. (C) 2009 Optical Society of America

OCIS codes: $090.1760,180.6900,100.3190,100.3020,110.1758$.

Optical scanning holography (OSH) compresses the $3 \mathrm{D}$ intensity information of an object into a $2 \mathrm{D}$ hologram $[1,2]$. To interpret the compressed information, we need to reconstruct sectional images [3,4]. The conventional convolution method, Wiener filter, and Wigner distribution function have been employed to reconstruct the sectional images with some success in the rejection of defocus noise [5-7]. Recently we proposed an inverse imaging method and demonstrated its advantages for suppressing defocus noise and in reconstruction speed [8].

The existing inverse imaging technique, however, requires a priori knowledge of the Fresnel zone plates (FZPs) to correlate with the hologram to extract the desired sectional images. To achieve the reconstruction automatically, we propose a blind sectional image reconstruction algorithm that estimates the FZPs and performs the corresponding reconstruction to obtain sectional images with the rejection of defocus noise. The blind sectional image proposed here is also applicable to phase-shifting digital holography [9].

Given an object intensity $I_{0}(x, y, z)$, its complex hologram generated by OSH can be represented as [8]

$$
H(x, y)=\sum_{i=0}^{n} I_{0}\left(x, y, z_{i}\right) * \operatorname{FZP}\left(x, y, z_{i}\right),
$$

where $\operatorname{FZP}\left(x, y, z_{i}\right)=j / \lambda z_{i} \exp \left\{\left(-\pi / \mathrm{NA}^{2} z_{i}^{2}+j \pi / \lambda z_{i}\right)\left(x^{2}\right.\right.$ $\left.\left.+y^{2}\right)\right\}$ stands for the Gaussian-apodized FZP at $z_{i}$. The symbol $*$ denotes $2 \mathrm{D}$ convolution, and NA stands for the numerical aperture of the Gaussian function; $\lambda$ is the wavelength of the laser used to record the hologram.

Algebraically, the convolution operations can be replaced with a matrix multiplication using a lexicographical ordering [8]. We therefore convert $H(x, y)$ into the length- $N^{2}$ vector, $\gamma$, given that the hologram is of size $N \times N$. Also $I_{0}\left(x, y, z_{i}\right)$ will be converted into vectors and denoted $\psi_{i}$. Meanwhile, we form $N^{2} \times N^{2}$ matrices, $\mathrm{M}_{i}$, using values from $\operatorname{FZP}\left(x, y, z_{i}\right)$. Then Eq. (1) can be derived as

$$
\gamma=\sum_{i=0}^{n} \mathrm{M}_{i} \psi_{i}=\mathrm{M} \psi
$$

where $\mathrm{M}=\left[\mathrm{M}_{0} \ldots \mathrm{M}_{n}\right]$ and $\psi=\left[\psi_{0}^{T} \ldots \psi_{n}^{T}\right]^{T}$. In Eq. (2), $\mathrm{M}$ encapsulates the entire holographic recording process of the OSH system. Using the observed hologram $\gamma$ to derive the scanned object $\psi$ is an inverse problem. We solve it by the conjugate gradient method with nonnegativity constraint $[8,10]$.

The computation of such an inverse problem depends on the matrix, M. However, in reality we cannot know its exact content. The recovery of $\psi$ from $\gamma$ when $\mathrm{M}$ is not known is a blind recovery problem [11]. While in some cases the blind system is derived concurrent with the estimation of the unknown signal [12], here $M$ is generated from the FZPs corresponding to sections to be extracted in a hologram. Thus, an estimate of $z_{i}$ can be used to produce the desired FZPs and subsequently M.

The main question therefore is to find the correct depth parameters $z_{i}(i=0, \ldots, n)$ in the FZPs that correspond to the sectional images to be reconstructed [13]. Our technique is as follows. Suppose we convolve the hologram with the conjugate FZP at $z_{0}$. Using the matrix notation in Eq. (2), we have

$$
\mathrm{M}_{0}^{*} \gamma=\mathrm{M}_{0}^{*} \mathrm{M}_{0} \psi_{0}+\mathrm{M}_{0}^{*} \mathrm{M}_{1} \psi_{1}+\cdots+\mathrm{M}_{0}^{*} \mathrm{M}_{n} \psi_{n} .
$$

The first term on the right-hand side is real only, because the elements of the matrix multiplication originating from the convolution, $\operatorname{FZP}\left(x, y, z_{0}\right)$ $* \mathrm{FZP}^{*}\left(x, y, z_{0}\right)$, is a $\delta$ function and $\psi_{0}$ is an intensity function. Therefore $M_{0}^{*} M_{0} \psi_{0}$ is the desired recon- 
structed image plane at $z_{0}$. The other terms are complex and manifest as defocus noise or fringes. Tracking the imaginary part of Eq. (3), we have

$$
\begin{aligned}
\operatorname{Im}\left[M_{0}^{*} \gamma\right] & =\operatorname{Im}\left[M_{0}^{*} M_{0} \psi_{0}+M_{0}^{*} M_{1} \psi_{1}+\cdots+M_{0}^{*} M_{n} \psi_{n}\right] \\
& =\operatorname{Im}\left[M_{0}^{*} M_{1} \psi_{1}+\cdots+M_{0}^{*} M_{n} \psi_{n}\right] .
\end{aligned}
$$

Note that there is no contribution from the first term on the right-hand side, as $\mathrm{M}_{0}^{*} \mathrm{M}_{0} \psi_{0}$ is real.

On the other hand, suppose that the convolution is taken with a conjugate FZP at $z_{r}\left(z_{r} \neq z_{0}, \ldots, z_{n}\right)$; i.e., we reconstruct the plane at $z_{0}$ but with the wrong FZP. We refer to its matrix as $\mathrm{M}_{r}^{*}$. Then the imaginary part of the reconstruction is

$$
\operatorname{Im}\left[\mathrm{M}_{r}^{*} \gamma\right]=\operatorname{Im}\left[\mathrm{M}_{r}^{*} \mathrm{M}_{0} \psi_{0}+\cdots+\mathrm{M}_{r}^{*} \mathrm{M}_{n} \psi_{n}\right] .
$$

Comparing Eq. (4) with Eq. (5), we note that the use of an incorrect FZP to reconstruct the sectional intensity at $z_{0}$ generates extra fringes on the $z_{0}$ plane, namely, the extra term $\mathrm{M}_{r}^{*} \mathrm{M}_{0} \psi_{0}$ in Eq. (5), assuming that the fringes originating from $\mathrm{M}_{k} \psi_{k}$ are almost the same for the two equations, where $k \neq 0$. To quantify their amount, we perform edge detection on the imaginary part of the convolution and refer to the total number of edge pixels as the edge amount. To detect the correct depth, we convolve the hologram with a series of FZPs with the depth parameter distributed uniformly. With a plot showing the edge amount corresponding to different depth distances, the local minima in the curve suggest where sections in the hologram contain the correct depth for reconstruction.

We demonstrate the performance of the blind sectional image reconstruction on experimental data in Fig. 1. The system is composed of a Mach-Zehnder interferometer and an electronic processing unit. A beam splitter (BS1) divides the laser beam into upper- and lower-path beams. The upper path generates a spherical wave through beam expander BE1 and lens L1 toward the object. The frequency of the spherical wave is upshifted by $\Omega+\Delta \Omega$ through acousto-optical frequency shifter AOFS1. The lower path generates a plane wave through $\mathrm{BE} 2$, which is upshifted in frequency by $\Omega$ through AOFS2.

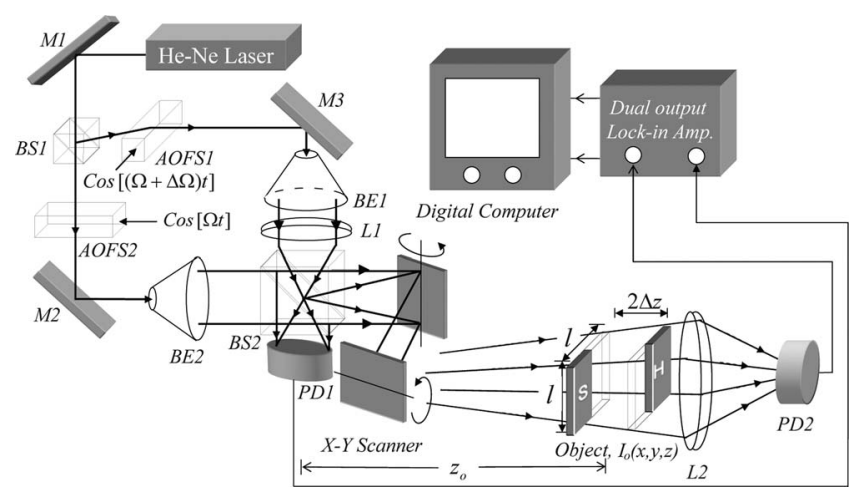

Fig. 1. OSH system. M, mirrors; AOFS1, AOFS2, acoustooptical frequency shifters; BS1, BS2, beam splitters; BE1, BE2, beam expanders; L1, focusing lens; L2, collecting lens; PD1, PD2, photodetectors.
The spherical wave and the plane wave are combined at the second beam splitter (BS2) to generate a time-dependent FZP-to-2D scan over the object [4]. In the experiment, $(\Omega+\Delta \Omega) / 2 \pi=40 \mathrm{MHz}$ and $\Omega / 2 \pi$ $=40.01 \mathrm{MHz}$, and hence a heterodyne current at a frequency of $\Delta \Omega / 2 \pi=10 \mathrm{kHz}$ is generated at the output of photodetector PD2 after collecting lens L2. The electronic processing unit is a dual output lock-in amplifier, which accepts the heterodyne current from photodetector PD1 as a reference signal. The lock-in amplifier then demodulates the signal from PD2 to give two holograms to be stored in a digital computer. The two holograms are subsequently added in a complex manner to give a complex hologram of the form given by Eq. (1) [4].

In the experiment, $z_{0}=87 \mathrm{~cm}$ and $\Delta z=10 \mathrm{~cm}$. The diameter of the collimated beam is $D=25 \mathrm{~mm}$, and the focal length of the lens is $f=500 \mathrm{~mm}$. Thus, the NA of the recorded full-parallax complex hologram is $\mathrm{NA} \approx D /(2 f)=0.025$. Shown in Fig. 2 are the real and imaginary parts of the complex hologram recorded by the OSH system, where the object consists of two transparencies of the letters "S" and " $\mathrm{H}$," with "S" located at $z_{0}$ and " $\mathrm{H}$ " at $z_{0}+2 \Delta z$ away from BS2.

To recover the sections, the blind sectional image reconstruction is implemented. We determine the FZPs associated with the sections first. The hologram is convolved with a sequence of FZPs with the depth parameter varying uniformly between 79.63 and $113.32 \mathrm{~cm}$ from the $x-y$ scanner. The Prewitt edge detector evaluates the edge amounts from Eqs. (4) and (5). Figures 3(a) and 3(b) show the output for two planes at $z=83.83 \mathrm{~cm}$ and $z=86.98 \mathrm{~cm}$ based on Eqs. (4) and (5). Figures 3(c) and 3(d) show the edge detection. We see that the edge amount is smaller when the plane is in focus, which is at $87 \mathrm{~cm}$ for "S." In Fig. 4 , we plot the edge amount in the imaginary part of the $n$th convolution result (the $y$ axis) versus the $x$ axis, which denotes the $n$th convolution at the distance $[79.63+(n-1) 1.05] \mathrm{cm}$. Two dominant troughs found in the plot illustrate that the FZPs of the reconstructed sections are at 87 and $107 \mathrm{~cm}$. Note that random noise may introduce additional small troughs, which is the case in Fig. 4. We can either suppress them by smoothing the curve (with the smoothness dependent on the noise amount) or simply reconstruct for more planes and discard them when it is later determined that there is no useful information at that focus plane.

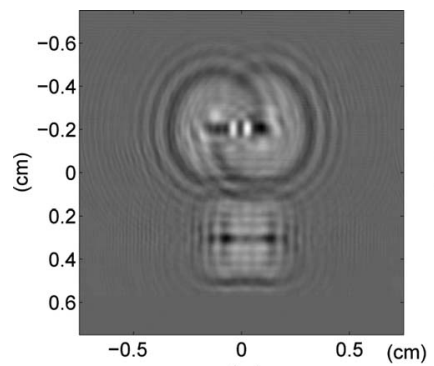

(a)

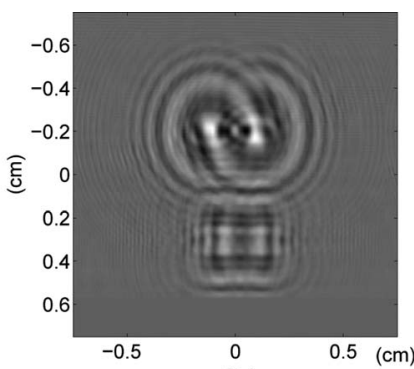

(b)
Fig. 2. (a) Real and (b) imaginary parts of the recorded hologram. 


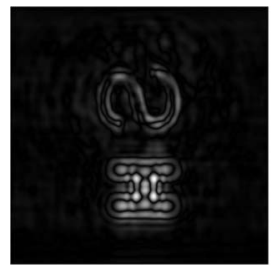

(a)

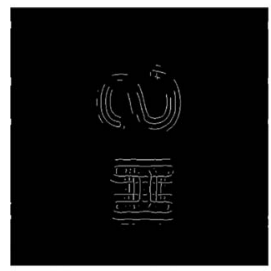

(c)

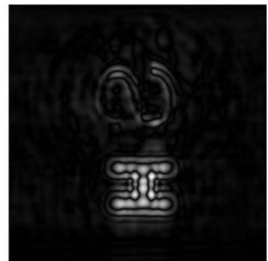

(b)

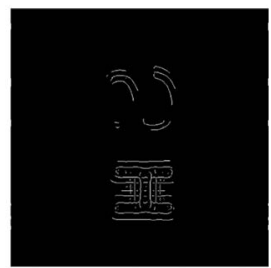

(d)
Fig. 3. Imaginary parts of convolution results and their edge detection results. (a) Imaginary result with FZP at depth distance of $83.83 \mathrm{~cm}$, (b) Same as (a) but at $86.98 \mathrm{~cm}$. (c) Edge detection of (a), and (d) edge detection of (b). Note that at $87 \mathrm{~cm}$, the "S" should be in focus. It is clear that at around $87 \mathrm{~cm}$, as shown in (d), the edge amount is smaller than that in (c).

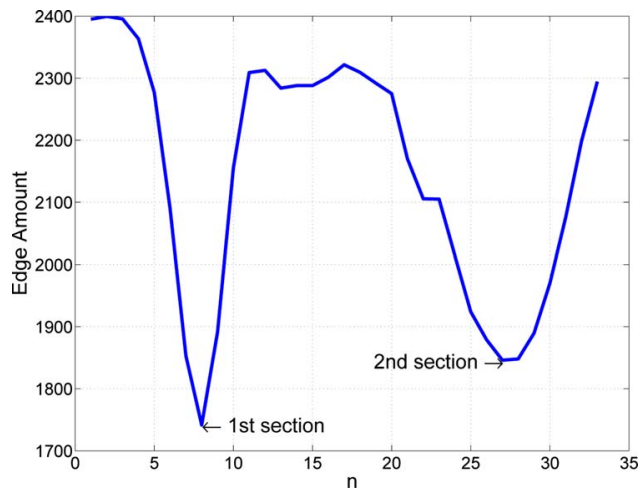

Fig. 4. (Color online) Plot of the edge amount (scale arbitrary) of a sequence of convolutions.

FZPs with the extracted depth parameters are then used in the inverse imaging algorithm [8]. To illustrate the performance, we compare the reconstructed images with those obtained by the conventional convolution method [5]. Shown in Fig. 5 are

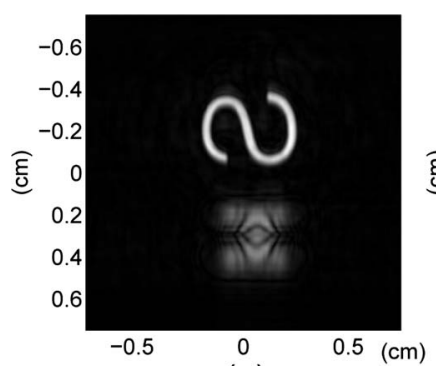

(a)

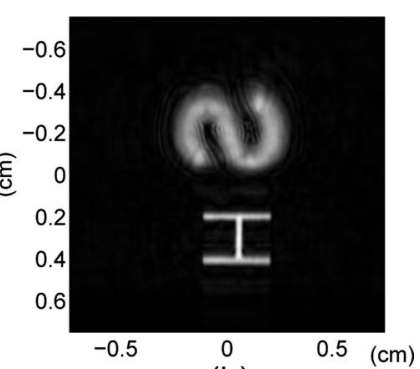

(b)
Fig. 5. Sectional images reconstructed by the convolution method.

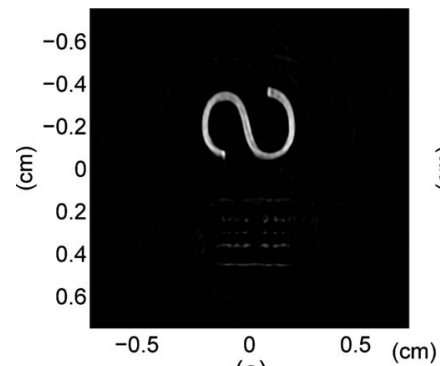

(a)

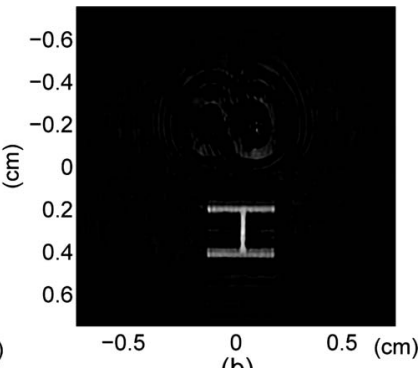

(b)
Fig. 6. Sectional images reconstructed by inverse imaging.

reconstructed images obtained by the latter method. In the two sectional images, the focused elements ("S" in the first image and " $\mathrm{H}$ " in the second one) are clearly reconstructed. We can also observe the defocus noise originating from the interference in sections adjacent to the focused section.

Our blind sectional image reconstruction uses the inverse imaging reconstruction to obtain sectional images. The FZPs computed by the edge detection method are used to render the matrix, M. Shown in Fig. 6 are two recovered sectional images. There is little defocus noise in the reconstructed images, and the focused parts of "S" and " $\mathrm{H}$ " are recovered in their respective images. In addition, we also perform the reconstruction on a simulated hologram with five focused sections (data accessible at http:// www.eee.hku.hk/isl). The blind sectioning method can also retrieve the right positions for reconstruction.

In conclusion, the blind sectional image reconstruction is proposed to recover sectional images automatically. The reconstruction is verified on real hologram data obtained from an OSH system.

\section{References}

1. T.-C. Poon, J. Opt. Soc. Am. A 2, 521 (1985).

2. J. Swoger, M. Martínez-Corral, J. Huisken, and E. Stelzer, J. Opt. Soc. Am. A 19, 1910 (2002).

3. E. Lam, X. Zhang, H. Vo, T.-C. Poon, and G. Indebetouw, "Three-dimensional microscopy and sectional image reconstruction using optical scanning holography," Appl. Opt. (Doc. ID 114194, to be published).

4. T.-C. Poon, J. Hologr. Speckle 1, 6 (2004).

5. T.-C. Poon and P. Banerjee, Contemporary Optical Image Processing with MATLAB, 1st ed. (Elsevier, 2001).

6. T. Kim, Appl. Opt. 45, 872 (2006).

7. H. Kim, S.-W. Min, B. Lee, and T.-C. Poon, Appl. Opt. 47, 164 (2008).

8. X. Zhang, E. Y. Lam, and T.-C. Poon, Opt. Express 16, 17215 (2008).

9. J.-P. Liu and T.-C. Poon, Opt. Lett. 34, 250 (2009).

10. C. R. Vogel, Computational Methods for Inverse Problems, 1st ed. (SIAM, 2002).

11. E. Y. Lam and J. W. Goodman, J. Opt. Soc. Am. A 17, 1177 (2000).

12. Z. Xu and E. Y. Lam, Opt. Lett. 34, 1453 (2009).

13. F. Dubois, C. Schockaert, N. Callens, and C. Yourassowsky, Opt. Express 14, 5895 (2006). 